\title{
A Study on Bottom Friction Coefficient in the Bohai, Yellow, and East China Sea
}

\author{
Daosheng Wang, ${ }^{1}$ Qiang Liu, ${ }^{2}$ and Xianqing $\mathrm{Lv}^{1}$ \\ ${ }^{1}$ Laboratory of Physical Oceanography, Ocean University of China, Qingdao 266100, China \\ ${ }^{2}$ College of Engineering, Ocean University of China, Qingdao 266100, China \\ Correspondence should be addressed to Qiang Liu; liuqiang@ouc.edu.cn
}

Received 9 April 2014; Revised 10 June 2014; Accepted 12 June 2014; Published 1 July 2014

Academic Editor: Fatih Yaman

Copyright (C) 2014 Daosheng Wang et al. This is an open access article distributed under the Creative Commons Attribution License, which permits unrestricted use, distribution, and reproduction in any medium, provided the original work is properly cited.

\begin{abstract}
The adjoint tidal model based on the theory of inverse problem has been applied to investigate the effect of bottom friction coefficient (BFC) on the tidal simulation. Using different schemes of BFC containing the constant, different constant in different subdomain, depth-dependent form, and spatial distribution obtained from data assimilation, the $\mathrm{M}_{2}$ constituent in the Bohai, Yellow, and East China Sea (BYECS) is simulated by assimilating TOPEX/Poseidon altimeter data, respectively. The simulated result with spatially varying BFC obtained from data assimilation is better than others. Results and analysis of BFC in BYECS indicate that spatially varying BFC obtained from data assimilation is the best fitted one; meanwhile it could improve the accuracy in the simulation of $\mathrm{M}_{2}$ constituent. Through the analysis of the best fitted one, new empirical formulas of BFC in BYECS are developed with which the commendable simulated results of $\mathrm{M}_{2}$ constituent in BYECS are obtained.
\end{abstract}

\section{Introduction}

The bottom friction plays a significant role in the tidal phenomenon. In numerical simulations of tide, bottom friction is generally parameterized by the bottom friction coefficient (BFC). In order to improve the simulation accuracy, it is essential to determine the $\mathrm{BFC}$ correctly. In previous studies [1-8], several methods were suggested to determine the BFC and some encouraging simulated results were achieved. Lee and Jung [9] used a three-dimensional mode-splitting, $\sigma$ coordinate barotropic finite-difference model to examine $\mathrm{M}_{2}$ tidal elevation and current in the Yellow Sea and East China Sea, and they treated the BFC as a constant in the whole computing domain. Zhao et al. [10] simulated the semidiurnal and diurnal tides and tidal currents in the whole Eastern China Seas with different BFC in different subdomain. Kang et al. [11] carried out a fine grid tidal modeling experiment to study the tidal phenomena in the Yellow and East China Seas, and they used the depthdependent form of BFC. He et al. [12] set up a numerical adjoint model with TOPEX/Poseidon (T/P) altimeter data to investigate the shallow water tidal constituents in the Bohai and Yellow Sea. In their model, the Bohai and Yellow Sea were divided into five sub-regions with different BFC. $\mathrm{Lu}$ and Zhang [13] used the adjoint method to assimilate T/P altimeter data into a 2-dimensional tidal model in the Bohai, Yellow, and East China Sea (BYECS) and the spatially varying $\mathrm{BFC}$ were estimated with the independent point strategy.

Additionally, open boundary conditions (OBCs) are crucial for the representation of tidal processes in the regional ocean model [14]. Generally, OBCs could be obtained from the larger scale model or by interpolating the existing observation data near the location. However, OBCs obtained by the methods mentioned above have to be adjusted by experience to get ideal simulated results. Based on the theory of inverse problem, the adjoint method is a powerful tool for parameter estimation [15], and thus OBCs could be optimized automatically. Zhang and $\mathrm{Lu}$ [16] applied the four-dimensional variational data assimilation technology to simulate the three-dimensional tidal currents in the marginal seas and the OBCs were optimized. Guo et al. [14] estimated the OBCs in Bohai Sea by an adjoint data assimilation approach with independent point strategy and obtained good simulated result of $\mathrm{M}_{2}$ constituent. Zhang and Wang [17] developed 
a new method based on the adjoint method to inverse the periodic OBCs in two-dimensional tidal models and used it to simulate the $\mathrm{M}_{2}$ constituent in BYECS successfully.

As mentioned above, BFC is an important parameter for tidal models and many schemes of BFC have been used in previous study. However, so far there are few systematic comparisons about the different schemes of BFC. Because different numerical models and observations are used in different studies, the simulated results in those papers in which the BFC are different could not be compared directly. In this paper, firstly the adjoint tidal model is employed to compare some different schemes of BFC. At the same time, in order to reduce the influence of OBCs that are also important for tidal models, we use the adjoint method to optimize OBCs. Based on the simulation of $\mathrm{M}_{2}$ constituent in BYECS, several different schemes of BFC including the constant, different constant in different subdomain, depthdependent form, and spatial distribution obtained from data assimilation are compared to find the best fitted one. Then we try to analyze the best fitted one to set up new empirical formulas of BFC in BYECS with which the preferable simulated results could be obtained.

\section{Adjoint Tidal Model}

2.1. Equations. The governing equations are described under the rectangular coordinate system. Assuming that pressure is hydrostatic and density is constant, the depth averaged twodimensional tidal model is as follows:

$$
\begin{array}{r}
\frac{\partial \zeta}{\partial t}+\frac{\partial[(h+\zeta) u]}{\partial x}+\frac{\partial[(h+\zeta) v]}{\partial y}=0 \\
\frac{\partial u}{\partial t}+u \frac{\partial u}{\partial x}+v \frac{\partial u}{\partial y}-f v+\frac{k u \sqrt{u^{2}+v^{2}}}{h+\zeta} \\
-A\left(\frac{\partial^{2} u}{\partial x^{2}}+\frac{\partial^{2} u}{\partial y^{2}}\right)+g \frac{\partial \zeta}{\partial x}=0 \\
\frac{\partial v}{\partial t}+u \frac{\partial v}{\partial x}+v \frac{\partial v}{\partial y}+f u+\frac{k v \sqrt{u^{2}+v^{2}}}{h+\zeta} \\
-A\left(\frac{\partial^{2} v}{\partial x^{2}}+\frac{\partial^{2} v}{\partial y^{2}}\right)+g \frac{\partial \zeta}{\partial y}=0
\end{array}
$$

where $t$ is time, $x$ and $y$ are Cartesian coordinates, $h$ is undisturbed water depth, $\zeta$ is sea surface elevation above the undisturbed sea level, $u$ and $v$ are velocity components in the east and north, $f$ is the Coriolis parameter, $g$ is the acceleration due to gravity, $k$ is the BFC, and $A$ is the horizontal eddy viscosity coefficient.

With the adjoint method described in Lu and Zhang [13], the cost function is constructed as

$$
J=\frac{1}{2} K_{\zeta} \int_{\Sigma}(\zeta-\widehat{\zeta})^{2} d \sigma
$$

where $K_{\zeta}$ is a constant and $\Sigma$ is the set of the observation locations.

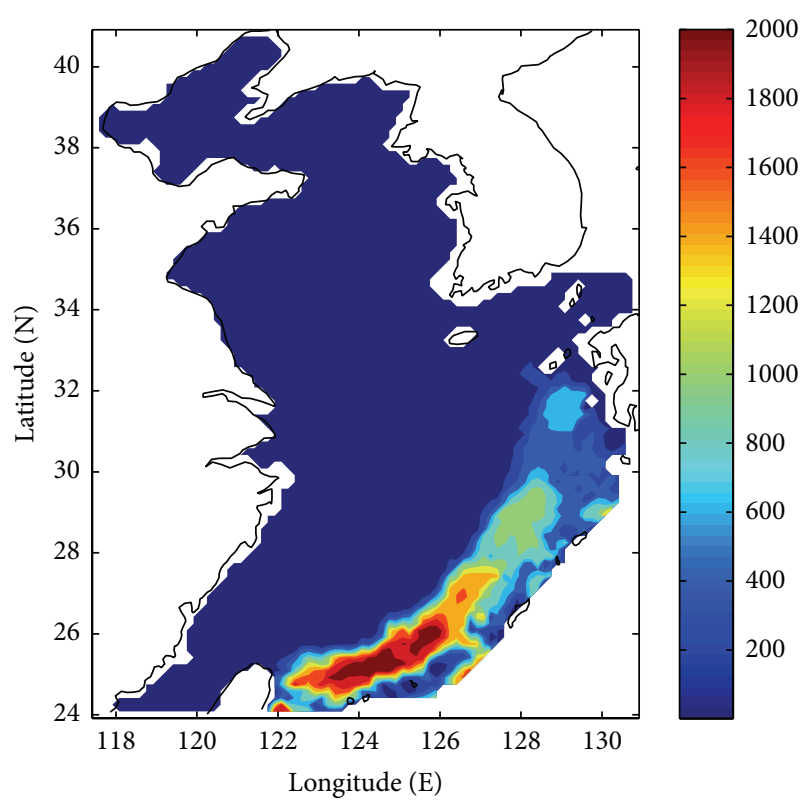

FIGURE 1: Bathymetry map of BYECS.

And the adjoint model can be constructed as follows:

$$
\begin{aligned}
\frac{\partial \lambda}{\partial t}+ & u \frac{\partial \lambda}{\partial x}+v \frac{\partial \lambda}{\partial y}+\frac{k \mu u \sqrt{u^{2}+v^{2}}}{(h+\zeta)^{2}}+\frac{k \nu v \sqrt{u^{2}+v^{2}}}{(h+\zeta)^{2}} \\
& +g \frac{\partial \mu}{\partial x}+g \frac{\partial v}{\partial y}=K_{\zeta}(\zeta-\widehat{\zeta}) \\
\frac{\partial \mu}{\partial t}- & \left(f+\frac{k u v}{(h+\zeta) \sqrt{u^{2}+v^{2}}}\right) v-\mu \frac{\partial u}{\partial x}-v \frac{\partial v}{\partial x} \\
& +\frac{\partial}{\partial x}(\mu u)+\frac{\partial}{\partial y}(\mu v)+(h+\zeta) \frac{\partial \lambda}{\partial x} \\
& +A\left(\frac{\partial^{2} \mu}{\partial x^{2}}+\frac{\partial^{2} \mu}{\partial y^{2}}\right)-\frac{k\left(2 u^{2}+v^{2}\right)}{(h+\zeta) \sqrt{u^{2}+v^{2}}} \mu=0 \\
\frac{\partial \nu}{\partial t}+ & \left(f-\frac{k u v}{(h+\zeta) \sqrt{u^{2}+v^{2}}}\right) \mu-\mu \frac{\partial u}{\partial y} \\
& -v \frac{\partial v}{\partial y}+\frac{\partial}{\partial x}(\nu u)+\frac{\partial}{\partial y}(\nu v)+(h+\zeta) \frac{\partial \lambda}{\partial y} \\
& +A\left(\frac{\partial^{2} v}{\partial x^{2}}+\frac{\partial^{2} v}{\partial y^{2}}\right)-\frac{k\left(u^{2}+2 v^{2}\right)}{(h+\zeta) \sqrt{u^{2}+v^{2}}} v=0
\end{aligned}
$$

where $\zeta$ is the simulated result, $\widehat{\zeta}$ is the observation, and $\lambda, \mu$, and $\nu$ denote the adjoint variables of $\zeta, u$, and $v$, respectively.

The finite difference schemes of (1) and (3) are similar to those in Lu and Zhang [13].

2.2. Model Setting. The computing area is BYECS $\left(117.5^{\circ} \mathrm{E}-131^{\circ} \mathrm{E}, 24^{\circ} \mathrm{N}-41^{\circ} \mathrm{N}\right)$ which is shown in Figure 1. The horizontal resolution is $10^{\prime} \times 10^{\prime}$. The time step is 62.103 


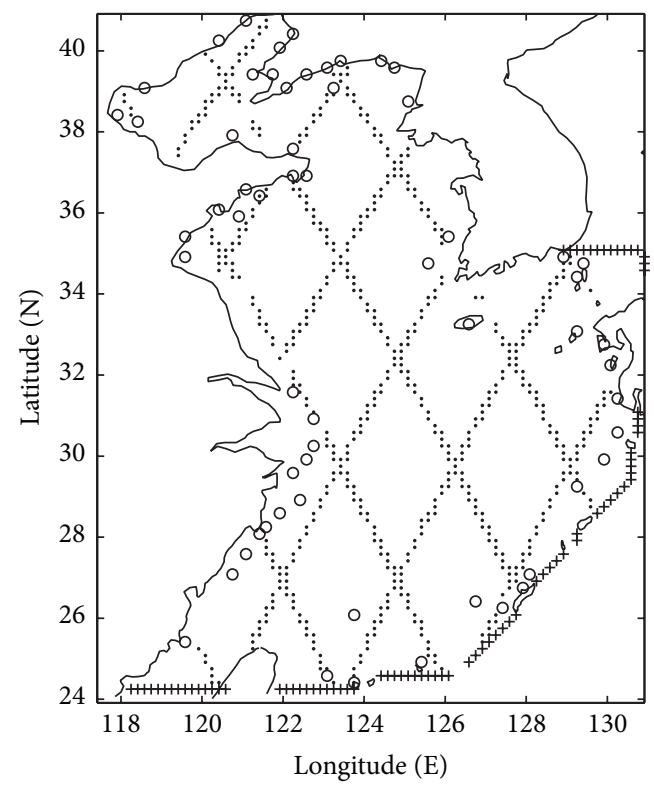

FIgure 2: Positions of T/P altimeter tracks (“•”) and tidal gauges (“o”) and open boundary (“+”).

seconds, which is $1 / 720$ of the period of $\mathrm{M}_{2}$ constituent. The eddy viscosity coefficient $(A)$ is $5000 \mathrm{~m}^{2} / \mathrm{s}$. The positions of tidal gauge stations, the T/P altimeter tracks, and the open boundary are shown in Figure 2.

\section{Numerical Experiments and Result Analysis}

3.1. Calculation Process of Numerical Experiments. Initial conditions are that the sea surface elevation $(\zeta)$ and the velocities $(u$ and $v)$ are zero. In addition, the initial values of OBCs are set to zero.

The calculation process of the adjoint tidal model is designed as follows.

(1) With the BFC given, which is fixed in the whole computing process, OBCs existed and other model parameters run the forward tidal model.

(2) The difference of water elevation between simulated results from step (1) and observations at the grid points on T/P satellite tracks serves as the external force of the adjoint model. Values of adjoint variables are obtained through backward integration of the adjoint equations.

(3) With the values of adjoint variables from the adjoint model, the OBCs could be adjusted by the method mentioned in Cao et al. [18].

Repeat steps (1)-(3) until the number of iteration steps is exactly 100 . For the setting of adjoint tidal model in this study, 100 iteration steps are sufficient because both the cost function and the difference between observations and simulated results will decrease slowly after this step.

3.2. Setting of Numerical Experiments. In each numerical experiment, the BFC is fixed and the OBCs are optimized by

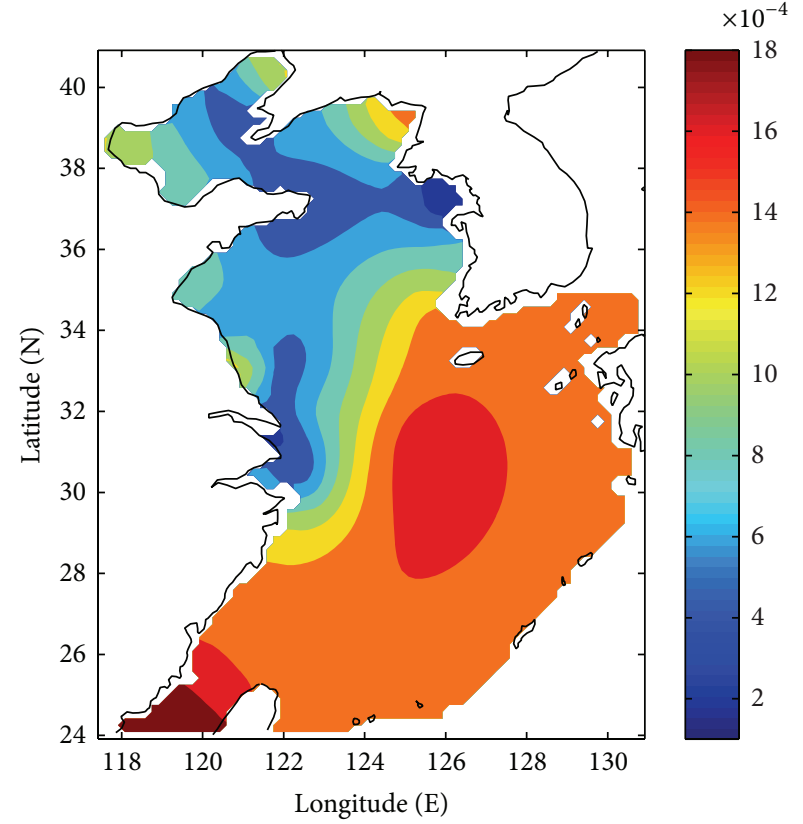

FIgURe 3: The BFC distribution in E4.

assimilating $\mathrm{T} / \mathrm{P}$ altimeter data into the adjoint tidal model, so that we could compare the different schemes of BFC adequately without the possibility that the OBCs do not match the BFC. Moreover, the tide gauge data are used as an independent check of the model fidelity.

Refer to some schemes of BFC generally used in previous studies, and we design several numerical experiments to compare them.

E1: the BFC is treated as a constant (0.0015) in BYECS.

E2: the BFC is depth-dependent form which is similar to that used by Kang et al. [11]. The BFC is defined by $k=g / C^{2}$, where $g$ is gravity acceleration, $C$ is Chezy coefficient, and the depth-dependent form of the Chezy coefficient are applied as $C=h^{1 / 6} / n$ with $n=0.023$.

E3: the scheme of BFC is the same as that employed in Zhao et al. [10]. The BFC is taken to be 0.001 at the west of the line from $\left(25^{\circ} 15^{\prime} \mathrm{N}, 120^{\circ} 45^{\prime} \mathrm{E}\right)$ to $\left(40^{\circ} 00^{\prime} \mathrm{N}\right.$, $\left.124^{\circ} 15^{\prime} \mathrm{E}\right), 0.0035$ in the Korean Strait, and 0.0016 in other areas.

E4: the space-varying BFC is obtained by assimilating observations using the adjoint method in $\mathrm{Lu}$ and Zhang [13]. The difference is that the initial condition of $\mathrm{BFC}$ in this paper is 0.0015 . The spatial distribution of BFC is shown in Figure 3.

3.3. Results of Numerical Experiments. When the tide is stable, the results of next period are used to do harmonic analysis. The mean absolute errors (MAEs) in amplitude and phase between simulation results and observations $(\mathrm{T} / \mathrm{P}$ data and tidal gauge data) are shown in Table 1. 
TABLE 1: Differences between simulated results and observations ( $\mathrm{T} / \mathrm{P}$ data and tidal gauge data).

\begin{tabular}{lcccc}
\hline EXP & \multicolumn{2}{c}{ MAEs of T/P data } & \multicolumn{2}{c}{ MAEs of tidal gauge data } \\
& $\begin{array}{c}\text { Amplitude } \\
(\mathrm{cm})\end{array}$ & Phase lag $\left(^{\circ}\right)$ & $\begin{array}{c}\text { Amplitude } \\
(\mathrm{cm})\end{array}$ & Phase lag $\left(^{\circ}\right)$ \\
\hline E1 & 7.2 & 6.2 & 10.2 & 7.3 \\
E2 & 7.6 & 6.7 & 10.3 & 8.9 \\
E3 & 6.9 & 6.1 & 9.9 & 7.2 \\
E4 & 5.7 & 5.8 & 6.7 & 6.6 \\
\hline
\end{tabular}

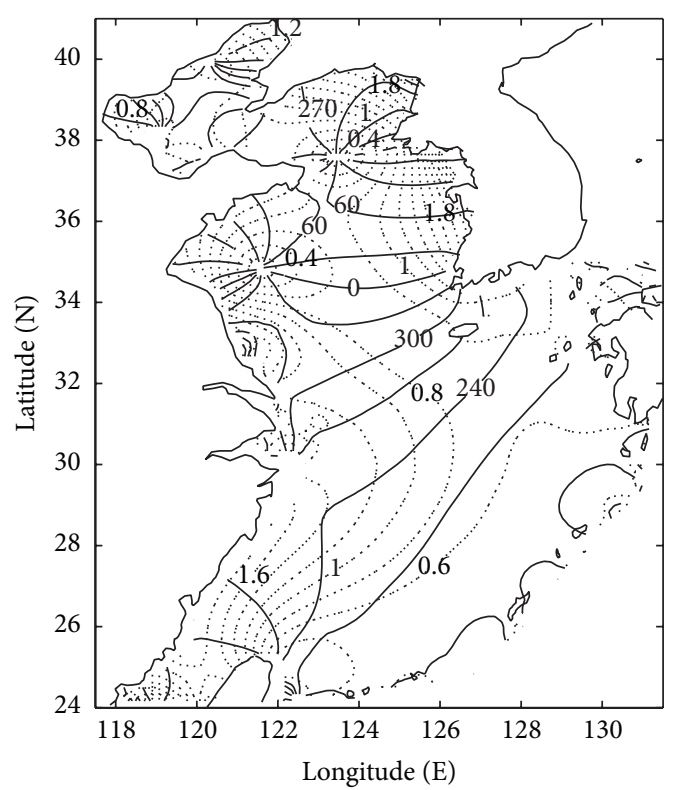

FIgURE 4: The cotidal chart obtained from E4 (the dashed line denotes coamplitude line (m), and solid line denotes cophase line (degree)).

From Table 1, one can find that E4 obtains the best simulated result. From the MAEs in amplitude and phase between simulation and T/P data, it could be found that E4 obtains the best assimilated results in the same steps of assimilation. And it is obvious that MAEs between simulation and tidal gauge data are minimum. We try to increase the number of iteration steps in E1, E2, and E3, but no improvements are achieved.

The cotidal chart of $\mathrm{M}_{2}$ constituent obtained in E4 is shown in Figure 4. Compared with Lefèvre et al. [19] and Fang et al. [20], the cotidal chart seems to coincide with the observed $\mathrm{M}_{2}$ constituent in BYECS fairly well. It also proves that E4 gets perfect simulated result. As shown in Figure 4, there are two amphidromic points in the Bohai Sea, one of which is near Qinhuangdao and the other is near the Yellow River delta. There are also two amphidromic points in the Yellow Sea, one of which is north of Chengshantou and the other is southeast of Qingdao.

\section{Discussion of BFC}

4.1. Discussion from Numerical Results. As shown by Table 1 and Figure 4, it is obvious that E4 with the space-varying BFC obtains the best simulated result.

Mofjeld [21] used a turbulence closure model to investigate the dependence on water depth of bottom stress and quadratic drag coefficient for a steady barotropic pressuredriven current in unstratified water when the current was the primary source of turbulence. He noted that the quadratic drag coefficient was approximated reasonably well by a formula from nonrotating channel theory in which the coefficient depended only on the ratio of the water depth to the bottom roughness. Jenter and Madsen [22] studied the bottom stress in wind-stress depth-average coastal flows and found that the drag tensor variation was a function of water depth, wind stress, and bottom roughness. From the aforementioned studies, it is seen that the BFC generally depends on the water depth and bottom roughness. And there is no doubt that the water depth and bottom roughness are diverse in different area and they vary spatially. In addition, Kagan et al. [23] studied the impact of the spatial variability in bottom roughness on tidal dynamics and energetics in the North European Basin and indicated that ignoring the spatial variability in bottom roughness was only partially correct because it was liable to break down for the tidal energetics. Therefore, the BFC should be spatially varying in fact. It is noticeable that the schemes of a constant BFC like in E1 is not reasonable enough. The space-varying BFC obtained from the data assimilation seems to be more advisable in physics.

In fact, BFC in E2 is depth-dependent, and thus it is also spatially varying. However, the simulated results from E2 are worse than that from E1 and E3 and much worse than that from E4. From Figure 3, the BFC in shallow water are larger than those in deep water in the Bohai Sea and the Yellow Sea individually. Meanwhile, the average water depth of the Bohai Sea is $19.3 \mathrm{~m}$ and the average BFC is 0.00082 , while they were $45.4 \mathrm{~m}$ and 0.00081 for the Yellow Sea and $334.7 \mathrm{~m}$ and 0.0015 for the East China Sea. From the definition of BFC in E2, it is evident that the $\mathrm{BFC}$ and the depth are in inverse proportion in whole region. In detail, the average BFC of the Bohai Sea is 0.0021 , while it is 0.0017 for the Yellow Sea and 0.0011 for the East China Sea. We can find that the BFC in the Bohai Sea and the Yellow Sea has the same changing trend with E4, but the value is larger. Green and McCave [24] indicated that the form drag caused by the bottom topography, wavecurrent interaction, boundary-layer stratification, and so on may impact the BFC. The water depth changes largely in the Okinawa trough, so the form drag should be larger. But in $\mathrm{E} 2$ the BFC in East China Sea is small and the East China Sea is the largest area in BYECS, so the simulated result of E2 is dissatisfactory. We surmise that the scheme of BFC in E2 may be reasonable in the shelf sea and not applicable in the area of slope and trough. Thus, it can be seen that the spatially varying BFC from data assimilation is better than the depthdependent form in BYECS, especially in the East China Sea.

In addition, E3 obtains better result than E2. In E2, the BFC is 0.0019 at the west of the line in E3, 0.0012 in the Korean Strait, and 0.0014 in other areas; meanwhile they are 0.0008 , 


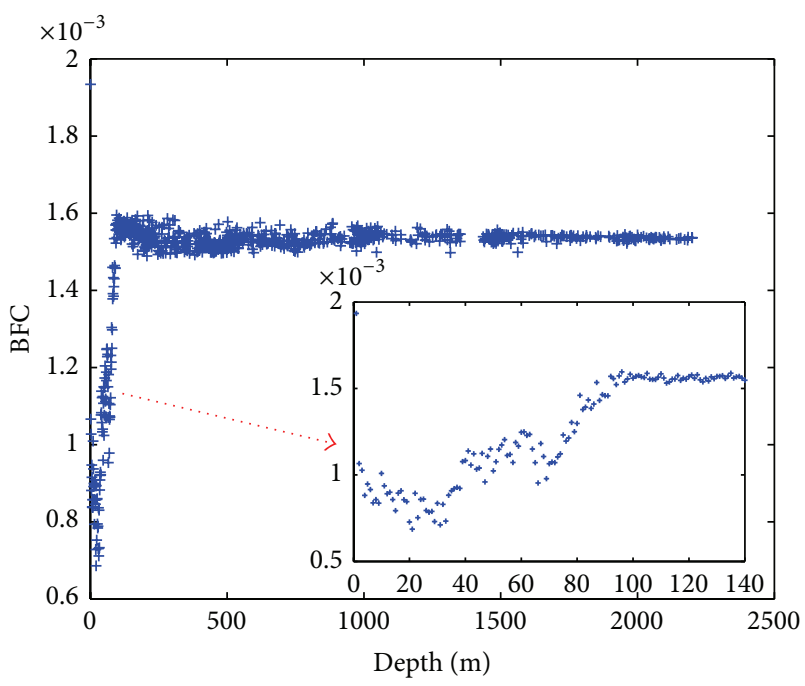

FIGURE 5: BFC versus water depth.

0.0014, and 0.0015 in E4. In the areas except the Korean Strait whose area is small, the BFC in E3 and E4 have the same changing tendency and the average values are approximately equal. However there is the opposite trend in E2. It seems to explain that the BFC in E3 is better than that in E2. And it proves that the scheme of BFC in E4 is the best fitted one from another side.

In conclusion, the spatially varying BFC in $\mathrm{E} 4$ is the best fitted BFC in BYECS.

4.2. Further Exploration of BFC. In this section, the scheme of $\mathrm{BFC}$ in $\mathrm{E} 4$ is analyzed to investigate the relationship between $\mathrm{BFC}$ and water depth, the change rate of seafloor topography (CRST), and bottom roughness.

In this study, CSRT is described as follows:

$$
\begin{aligned}
D h= & \left|h_{i, j}-h_{i+1, j}\right|+\left|h_{i, j}-h_{i-1, j}\right| \\
& +\left|h_{i, j}-h_{i, j+1}\right|+\left|h_{i, j}-h_{i, j-1}\right| .
\end{aligned}
$$

And bottom roughness is described as follows:

$$
\begin{aligned}
D D h= & \left(h_{i, j}-h_{i+1, j}\right)^{2}+\left(h_{i, j}-h_{i-1, j}\right)^{2} \\
& +\left(h_{i, j}-h_{i, j+1}\right)^{2}+\left(h_{i, j}-h_{i, j-1}\right)^{2} .
\end{aligned}
$$

The correlation coefficient between BFC and water depth is 0.4540 , while it is 0.3845 for CRST and 0.2520 for bottom roughness. It is shown that water depth is the significant factor that affects BFC. We demonstrate the BFC versus water depth in Figure 5 and could find that the BFC is a constant when water depth is larger than 100 meters. However, when water depth is less than 100 meters, the BFC varies complicatedly. So we focus on the study of quantitative relations of BFC with water depth, CRST and bottom roughness when water depth is less than 100 meters.

Considering BFC is mainly affected by water depth, in first step we ignore the CSRT and bottom roughness to make

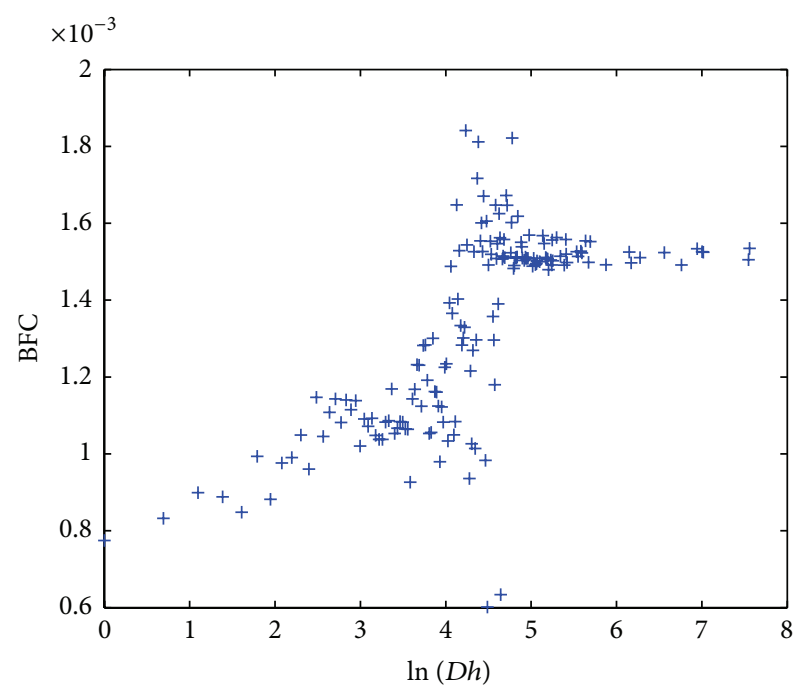

Figure 6: BFC versus $\ln (D h)$ when water depth is less than 100 meters.

the relationship simple and just investigate the quantitative relationship of BFC and water depth. From partial enlarged drawing in Figure 5, it could be seen that there are two sections. When water depth is less than 30 meters, BFC decreases with the water depth increasing, while increasing for larger than 30 meters. The fitting function could be obtained as follows:

$$
k= \begin{cases}1.5363 \times 10^{-3}, & h \geq 100 \\ (56.8850+0.9674 h) \times 10^{-5}, & 30 \leq h<100 \\ (100.0-0.5413 h) \times 10^{-5}, & h<30\end{cases}
$$

From another perspective, a linear function could describe the relationship between BFC and water depth roughly, and at the same time $D h$ and $D D h$ are also considered. As seen in Figure 6, BFC increases linearly along with $\ln (D h)$ by and large. From Figure 7 , it is shown that it is difficult to use a formula to describe the relationship between $\mathrm{BFC}$ and $\ln (D D h)$. Therefore considering the impact of $h, D h$ upon BFC, we obtain the formula as follows:

$k= \begin{cases}1.5363 \times 10^{-3}, & h \geq 100 \\ (0.5255+0.0068 h+0.0731 \ln (D h)) \times 10^{-3}, & h<100 .\end{cases}$

Using formulas (6) and (7), two new schemes of BFC in BYECS are obtained, and they are recorded as E5 and E6. The differences between simulated results and observations are shown in Table 2.

From Tables 1 and 2, it could be found that the simulated results of E5 and E6 are better than those of others except E4. It indicates that the schemes of BFC obtained from the statistical relation could describe the BFC in BYECS preferably and improve the result of numerical simulation.

Through the analysis of the scheme of BFC in E4, we set up new empirical formulas of BFC in BYECS with which the commendable simulated results are obtained. It should 


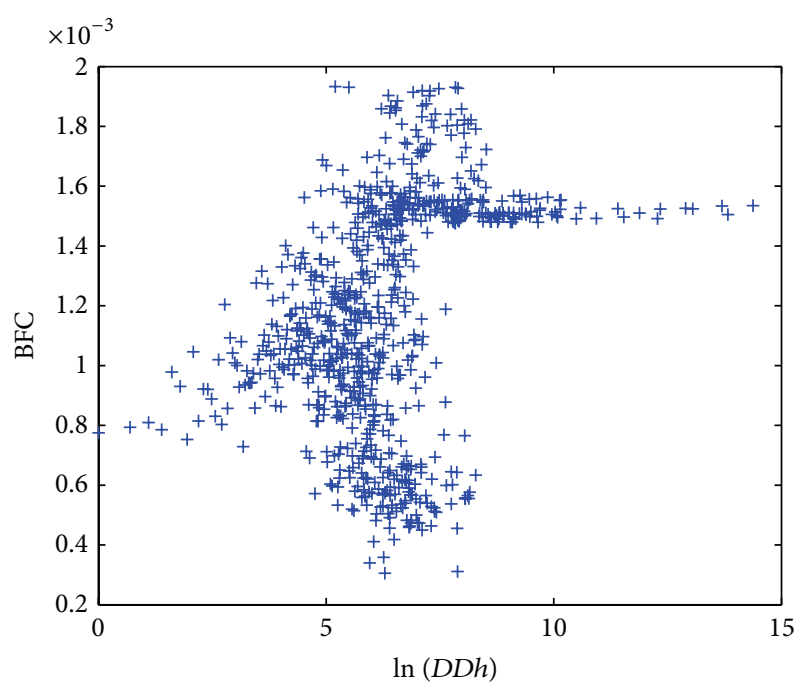

Figure 7: BFC versus $\ln (D D h)$ when water depth is less than 100 meters.

TABLE 2: Differences between simulated results and observations ( $\mathrm{T} / \mathrm{P}$ data and tidal gauge data).

\begin{tabular}{lcccc}
\hline EXP & \multicolumn{2}{c}{ MAEs of T/P data } & \multicolumn{2}{c}{ MAEs of tidal gauge data } \\
Amplitude & Phase lag $\left(^{\circ}\right)$ & $\begin{array}{c}\text { Amplitude } \\
(\mathrm{cm})\end{array}$ & Phase lag $\left(^{\circ}\right)$ \\
\hline E5 & 6.5 & 5.9 & 8.5 & 6.5 \\
E6 & 6.7 & 6.0 & 8.4 & 6.1 \\
\hline
\end{tabular}

be noted that the calculation of BFC in BYECS by the new empirical formulas just needs the bathymetric data. So it can be considered to be referenced in the simulation of $\mathrm{M}_{2}$ constituent in BYECS.

\section{Conclusions}

The adjoint tidal model based on the theory of inverse problem has been applied to investigate the effect of BFC on the tidal simulation. The $\mathrm{M}_{2}$ constituent in BYECS is simulated by assimilating $\mathrm{T} / \mathrm{P}$ altimeter data with several different schemes of BFC: the constant, different constant in different subdomain, depth-dependent form, and spatial distribution obtained from data assimilation. Comparing with the observations at tidal gauges, it is found that the simulated result with the spatially varying BFC is the best, and the MAEs in amplitude and phase are $6.7 \mathrm{~cm}$ and $6.6^{\circ}$, respectively, while the least values in other experiments are $9.9 \mathrm{~cm}$ and $7.2^{\circ}$. Comparing with the observations at $\mathrm{T} / \mathrm{P}$ stations, we found that the simulated result with spatially varying $\mathrm{BFC}$ has advantages over others and the MAEs in amplitude and phase are $5.7 \mathrm{~cm}$ and $5.8^{\circ}$, respectively, while in other experiments they are at least $6.9 \mathrm{~cm}$ and $6.1^{\circ}$. The simulated results and the analysis of BFC in BYECS simultaneously indicate that spatially varying BFC obtained from data assimilation is the best fitted one, and it could improve the accuracy in the simulation of $\mathrm{M}_{2}$ constituent. Finally, through the statistical analysis of the spatially varying
BFC obtained from data assimilation, new empirical formulas of BFC in BYECS are obtained. We found that the simulated results with new empirical formulas are better than traditional schemes, such as the constant, different constant in different subdomain, and depth-dependent form. We believe that the new empirical formulas could be referenced in the simulation of $\mathrm{M}_{2}$ constituent in BYECS.

\section{Conflict of Interests}

The authors declare that there is no conflict of interests regarding the publication of this paper.

\section{Acknowledgments}

The authors deeply thank the reviewers and editor for their constructive criticism of an early version of the paper. Partial support for this research was provided by the National Natural Science Foundation of China through Grants nos. 41072176 and 41371496, the National Science and Technology Support Program through Grant no. 2013BAK05B04, the State Ministry of Science and Technology of China through Grant no. 2013AA122803, and the Fundamental Research Funds for the Central Universities 201362033 and 201262007.

\section{References}

[1] C. Chen, H. Huang, R. C. Beardsley, H. Liu, Q. Xu, and G. Cowles, "A finite volume numerical approach for coastal ocean circulation studies: comparisons with finite difference models," Journal of Geophysical Research C: Oceans, vol.112, no. 3, Article ID C03018, 2007.

[2] L. S. Quaresma and A. Pichon, "Modelling the barotropic tide along the West-Iberian margin," Journal of Marine Systems, vol. 109-110, pp. S3-S25, 2013.

[3] J. Zhang and X. Lu, "Parameter estimation for a threedimensional numerical barotropic tidal model with adjoint method," International Journal for Numerical Methods in Fluids, vol. 57, no. 1, pp. 47-92, 2008.

[4] G. D. Egbert, R. D. Ray, and B. G. Bills, "Numerical modeling of the global semidiurnal tide in the present day and in the last glacial maximum," Journal of Geophysical Research C: Oceans, vol. 109, no. 3, Article ID C03003, 2004.

[5] H. J. Lee, K. T. Jung, J. K. So, and J. Y. Chung, "A threedimensional mixed finite-difference Galerkin function model for the oceanic circulation in the Yellow Sea and the East China Sea in the presence of $\mathrm{M}_{2}$ tide," Continental Shelf Research, vol. 22, no. 1, pp. 67-91, 2002.

[6] G. Sannino, A. Bargagli, and V. Artale, "Numerical modeling of the semidiurnal tidal exchange through the strait of Gibraltar," Journal of Geophysical Research C: Oceans, vol. 109, no. 5, 2004.

[7] A. W. Heemink, E. E. A. Mouthaan, M. R. T. Roest, E. A. H. Vollebregt, K. B. Robaczewska, and M. Verlaan, "Inverse 3D shallow water flow modelling of the continental shelf," Continental Shelf Research, vol. 22, no. 3, pp. 465-484, 2002.

[8] M. U. Altaf, M. Verlaan, and A. W. Heemink, "Efficient identification of uncertain parameters in a large-scale tidal model of the European continental shelf by proper orthogonal decomposition," International Journal for Numerical Methods in Fluids, vol. 68, no. 4, pp. 422-450, 2012. 
[9] J. C. Lee and K. T. Jung, "Application of eddy viscosity closure models for the $\mathrm{M}_{2}$ tide and tidal currents in the Yellow Sea and the East China Sea," Continental Shelf Research, vol. 19, no. 4, pp. 445-475, 1999.

[10] B. Zhao, G. Fang, and D. Cao, "Numerical modeling on the tides and tidal currents in the eastern China Seas," Yellow Sea Research, vol. 5, pp. 41-61, 1993.

[11] S. K. Kang, S. Lee, and H. Lie, "Fine grid tidal modeling of the Yellow and East China Seas," Continental Shelf Research, vol. 18, no. 7, pp. 739-772, 1998.

[12] Y. He, X. Lu, Z. Qiu, and J. Zhao, "Shallow water tidal constituents in the Bohai Sea and the Yellow Sea from a numerical adjoint model with TOPEX/POSEIDON altimeter data," Continental Shelf Research, vol. 24, no. 13-14, pp. 1521-1529, 2004.

[13] X. Lu and J. Zhang, "Numerical study on spatially varying bottom friction coefficient of a 2D tidal model with adjoint method," Continental Shelf Research, vol. 26, no. 16, pp. 19051923, 2006.

[14] Z. Guo, A. Cao, and X. Lv, "Inverse estimation of open boundary conditions in the Bohai Sea," Mathematical Problems in Engineering, vol. 2012, Article ID 628061, 9 pages, 2012.

[15] J. Zhang and H. Chen, "Semi-idealized study on estimation of partly and fully space varying open boundary conditions for tidal models," Abstract and Applied Analysis, vol. 2013, Article ID 282593, 14 pages, 2013.

[16] J. Zhang and X. Lu, "Inversion of three-dimensional tidal currents in marginal seas by assimilating satellite altimetry," Computer Methods in Applied Mechanics and Engineering, vol. 199, no. 49-52, pp. 3125-3136, 2010.

[17] J. Zhang and Y. Wang, "A method for inversion of periodic open boundary conditions in two-dimensional tidal models," Computer Methods in Applied Mechanics and Engineering, vol. 275, pp. 20-38, 2014.

[18] A. Cao, Z. Guo, and X. Lü, "Inversion of two-dimensional tidal open boundary conditions of $\mathrm{M}_{2}$ constituent in the Bohai and Yellow Seas," Chinese Journal of Oceanology and Limnology, vol. 30, no. 5, pp. 868-875, 2012.

[19] F. Lefèvre, C. le Provost, and F. H. Lyard, "How can we improve a global ocean tide model at a regional scale? a test on the Yellow Sea and the East China Sea," Journal of Geophysical Research C: Oceans, vol. 105, no. 4, pp. 8707-8725, 2000.

[20] G. Fang, Y. Wang, Z. Wei, B. H. Choi, X. Wang, and J. Wang, "Empirical cotidal charts of the Bohai, Yellow, and East China Seas from 10 years of TOPEX/Poseidon altimetry," Journal of Geophysical Research C: Oceans, vol. 109, no. 11, Article ID C11006, 2004.

[21] H. O. Mofjeld, "Depth dependence of bottom stress and quadratic drag coefficient for barotropic pressure-driven currents," Journal of Physical Oceanography, vol. 18, pp. 1658-1669, 1988.

[22] H. L. Jenter and O. S. Madsen, "Bottom stress in wind-driven depth-averaged coastal flows," Journal of Physical Oceanography, vol. 19, pp. 962-974, 1989.

[23] B. A. Kagan, E. V. Sofina, and E. Rashidi, "Inversion of twodimensional tidal open boundary conditions of $M_{2}$ constituent in the Bohai and Yellow Seas," Ocean Dynamics, vol. 62, no. 1012, pp. 1425-1442, 2012.

[24] M. O. Green and I. N. McCave, "Seabed drag coefficient under tidal currents in the eastern Irish Sea," Journal of Geophysical Research, vol. 100, no. 8, pp. 16057-16069, 1995. 


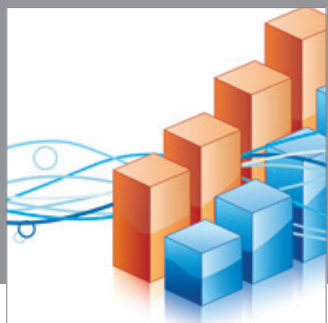

Advances in

Operations Research

mansans

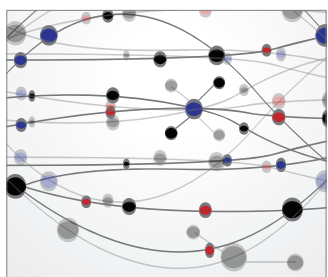

The Scientific World Journal
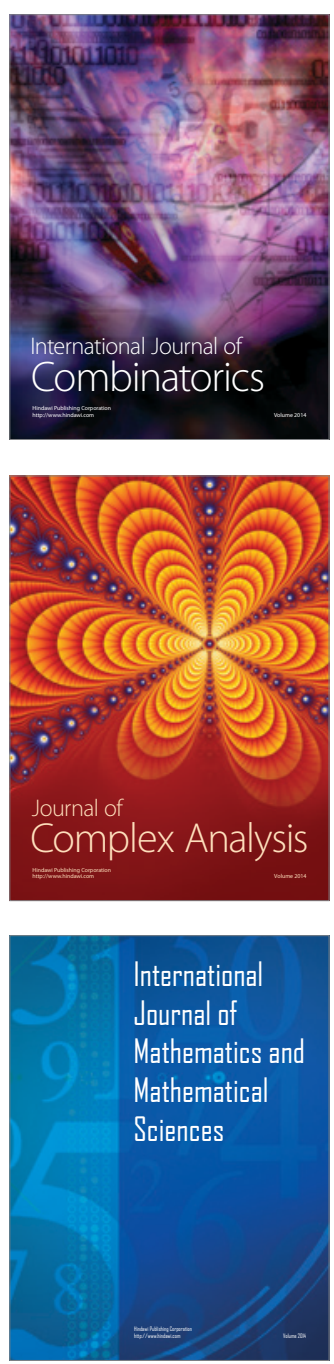
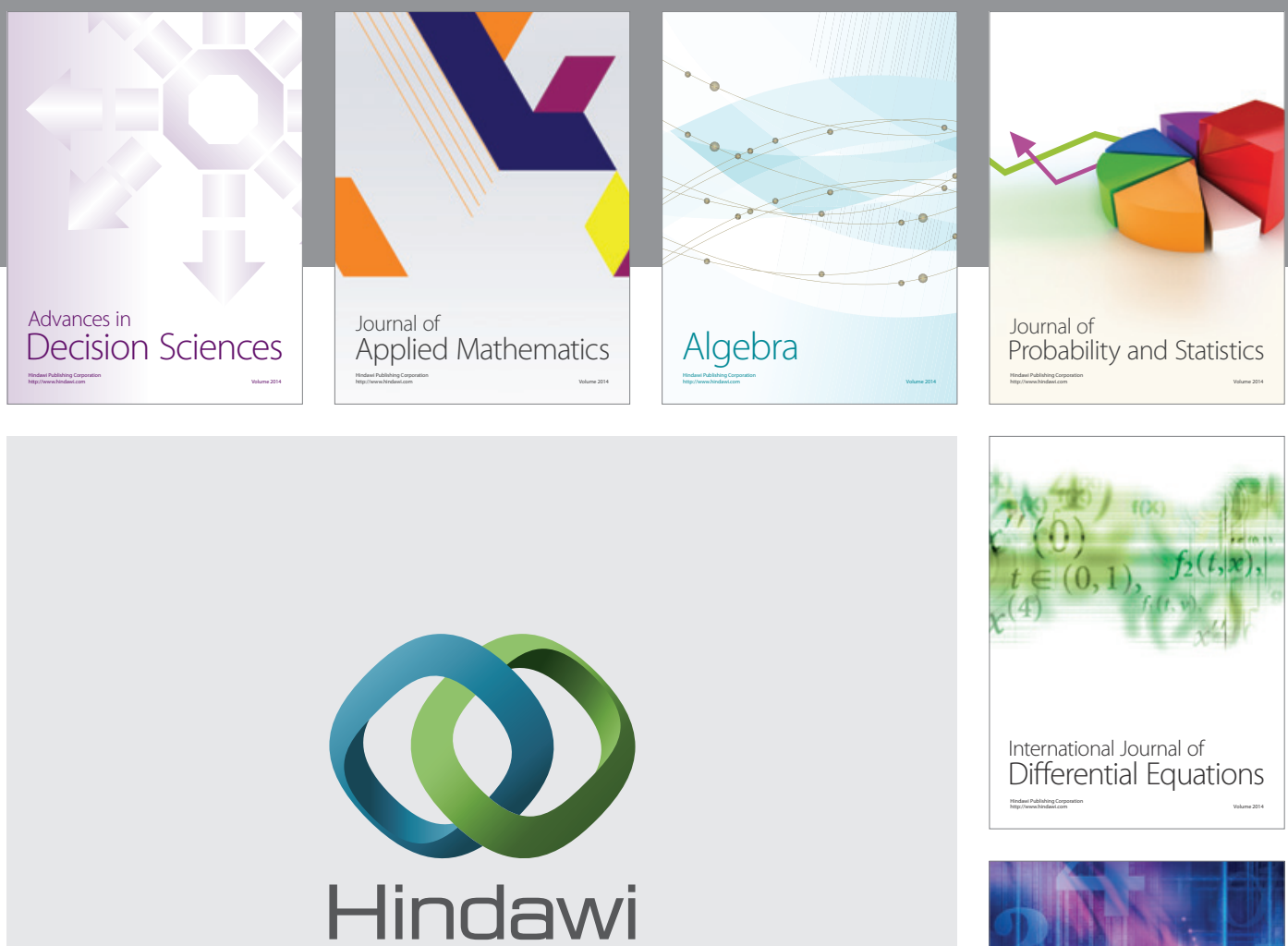

Submit your manuscripts at http://www.hindawi.com
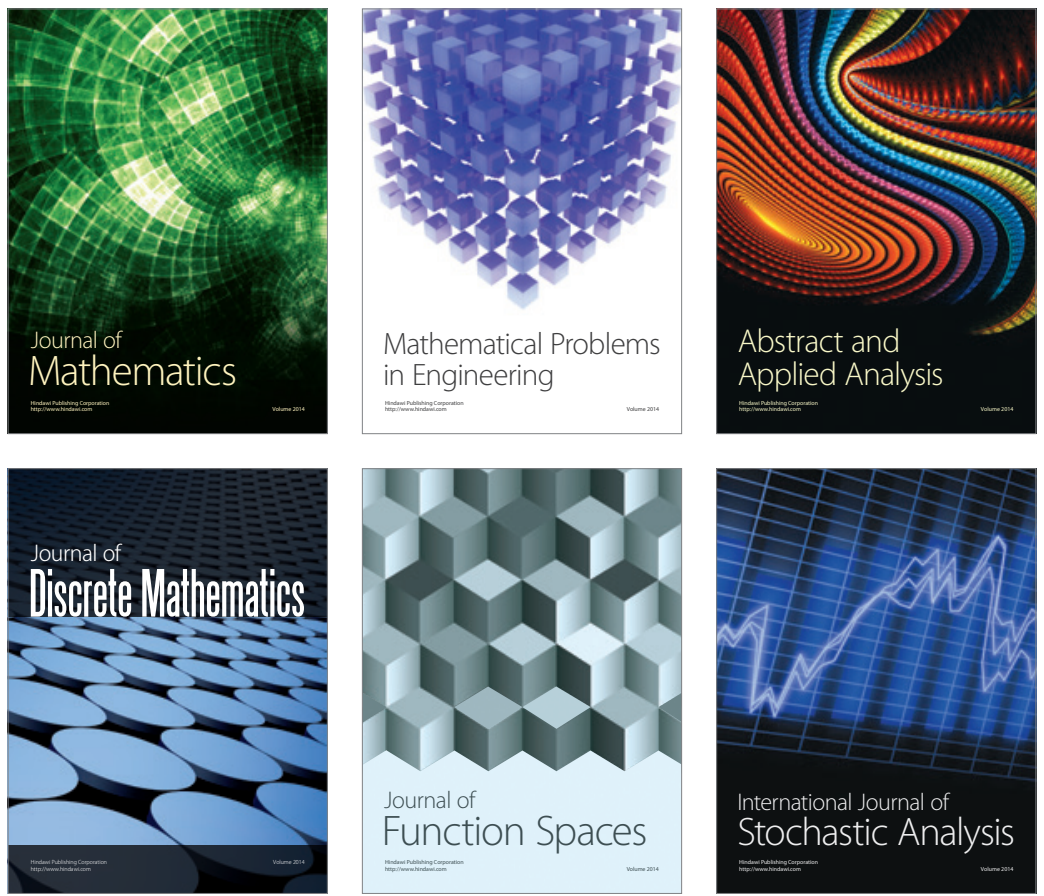

Journal of

Function Spaces

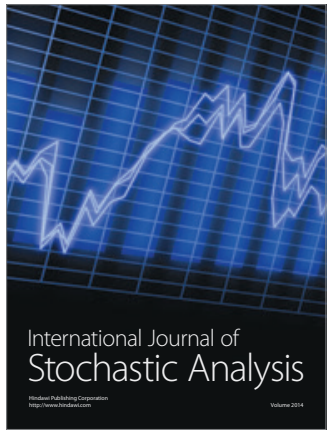

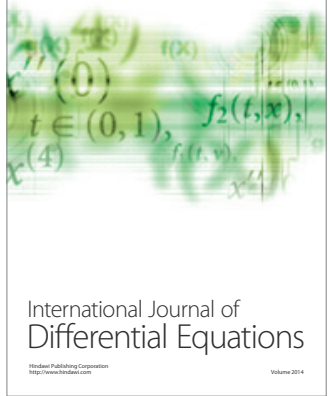
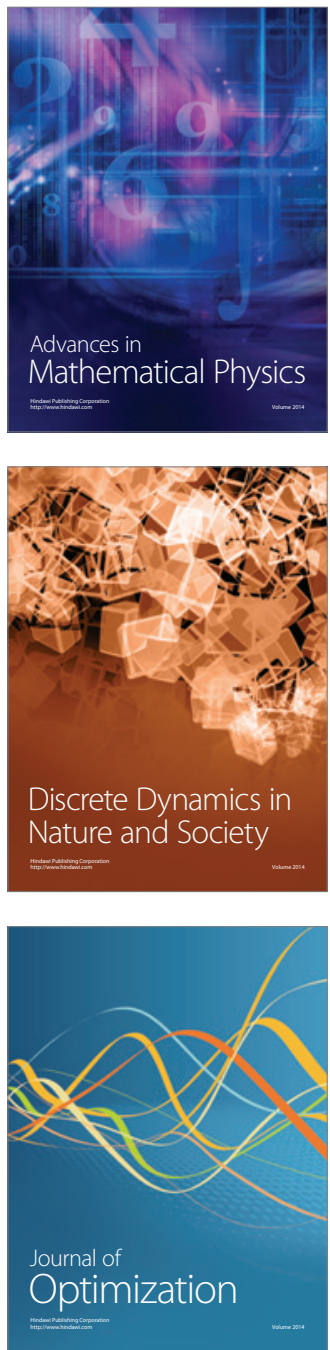